\title{
Medien-Frames als semantische Frames: Aspekte ihrer methodischen und analytischen Verschränkung am Beispiel der ,Snowden-Affäre'
}

\author{
Alexander Ziem, Christian Pentzold \& Claudia Fraas
}

\begin{abstract}
Der Beitrag stellt am Beispiel der sogenannten ,Snowden-Affäre' Möglichkeiten einer integrativen Frame-Analyse vor. Sie berücksichtigt sowohl linguistische, insbesondere diskurssemantische, Aspekte der Wissensrahmung als auch kommunikationswissenschaftliche Zugänge zur medialen Perspektivierung eines öffentlich brisanten Themas. Während ein diskurssemantischer Fokus Gefahr läuft, kommunikationsrelevante Faktoren wie Adressatenspezifik oder Bewertung auszublenden, mangelt es kommunikationswissenschaftlichen Untersuchungen von MedienFrames meist an der analytischen Orientierung an der sprachlichen Basis medialen Framings. Es ist das Ziel des Beitrages, den Ertrag einer paradigmenübergreifenden Verschränkung beider Perspektiven am Beispiel des Diskurses um die SnowdenAffäre in der US-amerikanischen Presse herauszuarbeiten und exemplarisch zu illustrieren.
\end{abstract}

\section{Einleitung: Frame-Analyse als disziplinübergreifendes Forschungsfeld}

Frames werden in unterschiedlichen Wissenschaftstraditionen zur Beschreibung von Wissensordnungen und deren Rolle in Verstehens- und Interpretationspro- 
zessen herangezogen. So arbeiten nicht nur kognitionswissenschaftliche und linguistische, sondern auch sozial- und insbesondere kommunikationswissenschaftliche Ansätze mit dem Konzept des Frames. ${ }^{1}$

Die begriffliche Klärung des Frame-Konzepts sowie sein analytischer Einsatz in empirischen Studien sind an die jeweiligen Erkenntnisinteressen der unterschiedlichen Forschungsrichtungen gebunden, was eine integrative Sicht der Ansätze erschwert, zumal mit demselben Begriffsinstrumentarium nicht notwendigerweise auf einen vergleichbaren Gegenstandsbereich zugegriffen wird (vgl. Ziem 2013: Abschnitt 2.1). Darüber hinaus werden neben dem Frame-Begriff auch andere Termini verwendet, um Wissensordnungen in Verstehens- und Interpretationsprozessen zu erfassen, etwa „schema“ (Tannen 1993), „script“ (Schank/ Abelson 1977), „scene“ (Fillmore 1977), „idealized cognitive model“ (Lakoff 1987) oder „domain“" (Fillmore 1982; Langacker 1987).

Vergleicht man die Frame-Ansätze in den Kognitions- und Sozialwissenschaften hinsichtlich ihrer grundsätzlichen Annahmen, so lassen sich bei aller Unterschiedlichkeit folgende Gemeinsamkeiten feststellen: Erstens gehen sie mehr oder weniger explizit davon aus, dass Frames sowohl im individuellen, kognitiven System verankert sind als auch stark durch überindividuelle soziale Prozesse beeinflusst werden. Zweitens werden Frames sowohl in kognitions- als auch in sozialwissenschaftlichen Ansätzen mit Kategorisierung, Perspektivierung, Selektion und Salienz in Verbindung gebracht. Drittens erfassen die kognitions- als auch die sozialwissenschaftlichen Ansätze Frames als Strukturen, die aus - wie auch immer gearteten - Frame-Elementen bestehen (vgl. Ziem 2013: Abschnitt 3.1).

Ausgehend von diesen grundlegenden fachübergreifenden Anknüpfungspunkten wäre es analytisch von Gewinn, die unterschiedlichen Frame-Konzepte der Forschungstraditionen zumindest in Teilen aufeinander zu beziehen, um Desiderata zu identifizieren, die einzelne Forschungsrichtungen im Kontext von Frame-Analysen jeweils betreffen (vgl. Fraas/Pentzold 2011; Ziem 2013). So kann,

1 Vgl. für die Kognitionswissenschaften Minsky 1975, 1977, 1988; Schank/Abelson 1977; Barsalou 1992; für linguistische Ansätze Busse 2012; Fillmore 1977, 1982, 1985; Konerding 1993; Fraas 1996; Löbner 2012; Lönneker 2003; Petersen 2007; Ziem 2008; für die Sozialwissenschaften Goffman 1977; Lüders/Meuser 1997; Plaß/Schetsche 2001; für Frame-Analysen im Rahmen der Theorie sozialer Bewegungen Gamson/Modigliani 1989; Benford/Snow 2000; für die Kommunikationswissenschaft Entman 1993; Scheufele 1999; Scheufele 2003; Dahinden 2006; Matthes 2007. 
wie im Folgenden argumentiert wird, die kommunikationswissenschaftliche Forschung von linguistischen framesemantischen Ansätzen zum einen hinsichtlich einer theoretisch-methodologischen Fundierung des Instrumentariums von Frame-Analysen profitieren. Denn der Frame-Begriff der Kommunikationswissenschaft fokussiert weniger den Strukturaspekt von Frames und die Frage, welcher konzeptuelle Status den angesetzten Frame-Elementen zukommt, sondern ist auf das Perspektivierungspotenzial von Frames in der (massen-)medial vermittelten Kommunikation ausgerichtet (Matthes 2007, 318). Das Konzept von Frames wird in der Kommunikationswissenschaft also auf Interpretationsschemata als kollektive Orientierungsmuster bezogen, die Wissensbestände perspektivieren und so bestimmte Informationen hervorheben und andere ausblenden. Zum anderen kann die kommunikationswissenschaftliche Rekonstruktion von MedienFrames analytisch von einer framelinguistischen Orientierung auf komplexe musterhafte Prädikationsstrukturen profitieren.

Das etablierte kommunikationswissenschaftliche Verständnis von Frames folgt der inzwischen klassischen Definition von Entman (1993, 52), nach der gilt: „to frame is to select some aspects of a perceived reality and make them more salient in a communicating context". Frames werden somit im weitesten Sinn als Sinnhorizonte verstanden, die Problemdefinitionen nahe legen, Ursachen ausmachen, eine Bewertung vornehmen und Lösungsmöglichkeiten aufzeigen. Trotz der wachsenden Anwendung von Frame-Analysen besteht in der Kommunikationswissenschaft über diese basalen Überlegungen hinaus kaum theoretischer und methodischer Konsens hinsichtlich der Analyse und Beschreibung von Frames (vgl. Hertog/McLeod 2001; Tankard 2001; Johnston 2002; Carragee/Roefs 2004; Matthes/Kohring 2008; Scheufele/Engelmann in diesem Band). Im Vergleich dazu erlaubt der framesemantische Ansatz - ausgehend von verbalen Elementen und darin ausgedrückten semantischen Strukturen - eine themenbezogen spezifischere Aufstellung von Frameelementen, um das Interpretationsrepertoire eines Diskurses zu erfassen. Die kognitiv orientierte linguistische Frame-Analyse wiederum könnte indessen von einer stärkeren Öffnung für Zeichenverwendungsprozesse profitieren, welche die kommunikationswissenschaftliche Frame-Forschung hinsichtlich des strategischen Einsatzes von Sprache und Bildern im Rahmen medialer Inszenierung im Blick hat. 
Um linguistische Perspektiven und kommunikationswissenschaftliche Anknüpfungspunkte für die Methodik und Praxis der Analyse von Medien-Frames aufzuzeigen, stellen wir zunächst den konzeptionellen Hintergrund diskurssemantischer Frame-Analysen vor und beziehen diesen methodisch auf Formen medialen Framings. Darauf aufbauend erläutern wir am Beispiel der so genannten Snowden-Affäre das Vorgehen einer Framing-Analyse von Schlüsselwörtern. Die exemplarisch genutzte mediale Berichterstattung der New York Times zur Snowden-Affäre bezieht sich auf die Aufdeckung geheimer Spionage- und Überwachungsmaßnahmen der USA und Großbritanniens durch den ehemaligen Geheimdienstmitarbeiter Edward Snowden Anfang Juni 2013 und der damit einhergehenden Veröffentlichung von Dokumenten der US-amerikanischen National Security Agency (NSA).

\section{Frame-Analyse: linguistische Perspektiven}

\subsection{Frames und Framing: konzeptioneller Hintergrund}

Den Gegenstandsbereich diskurssemantischer Frame-Analysen bildet der öffentliche Sprachgebrauch mit dem Ziel, sprachliche Konzeptualisierungen von verstehensrelevantem Wissen textanalytisch (etwa Holly 2001; Klein 2002) oder diskurslinguistisch zu analysieren (vgl. u. a. Fraas 1996; Ziem 2008; Busse 2012). ${ }^{2}$

Konzeptionell geht der linguistische Frame-Begriff auf Fillmores Studien zu „frames of understanding“ seit Mitte der 1970er Jahre zurück (etwa Fillmore 1975; 1985). ${ }^{3}$ Frames versteht Fillmore als konzeptuelle Strukturen, die den Gebrauch und das Verstehen von sprachlichen Ausdrücken motivieren. Um beispielsweise die Bedeutung des Verbes collect (,sammeln') im Kontext der Snowden-Affäre erfassen zu können, ist es notwendig, auf verstehensrelevante Konzepte zurückzugreifen, die im übergeordneten Wissensrahmen angelegt sind, so etwa Angaben dazu, was gesammelt wird, wer etwas zu welchem Zweck sammelt, an welchem

2 Vgl. hierzu die ausführlichere Darstellung in Ziem 2013 und Ziem 2017, worauf die folgenden konzeptionell-methodischen Erläuterungen teilweise basieren.

3 Neben Fillmores Ansatz war und ist darüber hinaus der von Minsky (1975) geprägte Frame-Begriff sowie Barsalous (1992) kognitionspsychologisch fundiertes Frame-Konzept überaus einflussreich, vgl. die Überblicke in Busse 2012, 251-439 und Ziem 2008, 13-58. Sie können für die weitere Argumentation aber vernachlässigt werden. 
Ort und zu welchem Zeitpunkt bzw. in welchem Zeitrahmen dies geschieht. Das linguistische Erkenntnisinteresse richtet sich auch auf jene Kenntnisse und (Hintergrund-)Annahmen, die nötig sind, um einen sprachlichen Ausdruck angemessen verwenden und verstehen zu können. Ziel ist es zu ermitteln, in welcher Gestalt verstehensrelevantes Wissen am Prozess der Bedeutungskonstitution beteiligt ist. Für das Schlüsselwort collect im Kontext der Snowden-Affäre heißt dies konkret, dass etwa Angaben zu Zweck, Umfang, Art und Weise und eingesetzten Hilfsmitteln eine herausragende Rolle spielen können.

Grundsätzlich sind Prozesse der Perspektivierung und Interpretation sowohl für die Linguistik als auch für die Kommunikationswissenschaft relevant und werden häufig mithilfe des Framing-Konzepts beschrieben, wenn auch auf unterschiedlichen Ebenen. Framing bezeichnet zum einen den Prozess der mehr oder weniger bewussten Aktivierung kognitiver Strukturen (vgl. Chong/Druckman 2007; Matthes 2007) und zum anderen die absichtsvolle Gestaltung von Botschaften (vgl. D’Angelo/Kuypers 2009). Entman, Matthes und Pellicano (2009) finden beispielsweise in ihrer an massenmedialen Kommunikationsverhältnissen orientierten Darstellung Framing-Prozesse (a) auf der Ebene von Medientexten und ihrer Herstellung, (b) aufseiten kommunizierender Eliten und politischer Kommunikatoren sowie (c) auf der Seite der Rezipienten, d. h. der Publika.

Während Frames Wissensstrukturen repräsentieren und modellieren, richtet sich der Begriff des Framing insbesondere auf den Prozess der Entstehung und Verfestigung dieser Strukturen (vgl. hierzu Klein in diesem Band). Dies ist prinzipiell in zweifacher Weise möglich: Erfolgt der Prozess der Rahmung intentional und ist er gesteuert durch strategische (etwa politische, ökonomische oder soziale) Interessen, so liegt ein Fall von „Begriffe besetzen“ (Liedtke/Wengeler/Böke 1991) vor. Wesentlich für diese Form des Framing ist das strategisch genutzte Zusammenspiel unterschiedlicher - sprachlicher, (audio-)visueller, akustischer Zeichenressourcen in kontextgebundenen Bedeutungskonstitutions- und Interpretationsprozessen und deren Gebrauch zur Kategorisierung, Perspektivierung und Selektion salienter Elemente einer Darstellung. Der moderne kognitions- und kommunikationswissenschaftliche Framing-Begriff orientiert sich an dieser Bestimmung (vgl. im politischen Kontext: Lakoff 2004; aus kommunikationswissenschaftlicher Sicht Entman 1993 und Matthes 2007). Begriffsprägungen infolge von 
Framing können aber auch das emergente Ergebnis des Sprachgebrauchs innerhalb einer Sprachgemeinschaft sein, ohne strategisch motiviert oder geplant zu sein. In diesem Fall spricht man in der Regel von „Begriffswandel“ (als einen Bereich des Sprachwandels, vgl. Keller 2003). Auch dieser erfolgt innerhalb von Diskursen und lässt sich von diesen nicht ablösen. ${ }^{4} \mathrm{Ob}$ strategisch-geplant oder unintendiert-emergent, für beide Fälle gilt, dass Framing-Prozesse gegenstandskonstitutiv sind, vergleichbar mit kommunikativen Rahmungen, wie Entman (1993) sie beschrieben hat.

Untersuchungen von Framing-Prozessen bieten sich etwa an, wenn Begriffe kontrovers diskutiert werden und mithin als strittig gelten. Einen Hinweis auf die öffentliche Brisanz eines Begriffes können metasprachliche Bezugnahmen geben, insofern sich an ihnen das Interesse zeigt, Bedeutungen bzw. Bedeutungsaspekte des in Frage stehenden Ausdrucks hervorzuheben oder gar zu fixieren (vgl. Stötzel 1995, 3 und 17; Stötzel 1980, 39; Wengeler 1996, 312 f.; zusammenfassend: Ziem 2008, 401 ff.). Zu den Aufgaben für die kommunikationswissenschaftlich ausgerichtete Framing-Forschung kann es in dieser Hinsicht gehören, lexikalischsemantischen Prägungen in spezifischen gesellschaftspolitischen Diskursen nachzuspüren und möglichst konzise zu erfassen. Bei dieser Aufgabe handelt es sich notwendigerweise um ein korpusbasiertes Unterfangen, zumindest dann, wenn über Einzelfallbeobachtungen hinaus fallübergreifende, generalisierende Aussagen getroffen werden sollen. Nur unter dieser Prämisse kann ein beobachtetes Phänomen als prototypisch oder musterhaft bewertet werden. ${ }^{5}$

Wie lässt sich Framing auf der lexikalischen Ebene datenbasiert identifizieren? Und welche Methoden stehen der sprach- und kommunikationswissenschaftlichen Forschung dabei zur Verfügung? Eine Möglichkeit, diskursive Prozesse der semantischen Musterbildung deskriptiv und kognitiv adäquat zu erfassen, bietet das Frame-Konzept, wie es dem Berkeleyer FrameNet-Projekt zugrunde liegt. Frames werden hier als konzeptuelle Strukturen behandelt, die das Verstehen und den Gebrauch sprachlicher Ausdrücke motivieren, indem sie einen begrifflichen

4 Vgl. am Beispiel des Begriffes Krise etwa Ziem/Scholz 2013.

5 Allein der Rückgriff auf exemplarische, typische Befunde und Beispiele erlaubt es, gesicherte Aussagen über das „Denken, Fühlen, Wollen“ (Hermanns 1995, 71 ff.) einer Kommunikationsgemeinschaft zu fällen. 
,Rahmen` für die Erfassung des jeweils relevanten Bedeutungsgehalts bereitstellen. Dieser Rahmen fungiert gleichsam als Konzeptualisierungsschablone, d. h. er macht Wissen über relevante, z. T. kulturspezifische Erfahrungen, Ereignisse einschließlich beteiligter Personen, Situationsparameter und des Hintergrundwissens - in strukturierter Weise verfügbar. In FrameNet geschieht dies durch so genannte Frame-Elemente, worunter jeweils framespezifisch definierte semantische Rollen zu verstehen sind (Fillmore/Baker 2010, 324-328). ${ }^{6}$ Lexikalische Bedeutungen lassen sich folglich mit Blick auf den Frame, den sie aufrufen, bestimmen, wobei jedes Lexem die im Frame angelegten semantischen Rollen je spezifisch konkretisiert. Die Ausgestaltung der komplexen musterhaften Prädikationsstrukturen variiert zudem in Abhängigkeit vom Ko- und Kontext, in dem die lexikalische Einheit eingebettet ist. Sowohl einzelne Frame-Elemente bzw. Leerstellen als auch spezifische Realisierungen bzw. Füllungen derselben (Prädikate) können sich in einer Sprachgemeinschaft oder in Diskursen verfestigen und so zu spezifischen Bedeutungsprägungen führen.

Vor diesem konzeptionellen Hintergrund können Frames einerseits als kognitives Repräsentationsformat von semantischem Wissen dienen, andererseits aber auch als Werkzeug zur Analyse von sprachlichen Bedeutungen eingesetzt werden. In Fillmores $(1985,232)$ Worten: „In addition to seeing frames as organizers of experience and tools for understanding, we must also see frames as tools for the description and explanation of lexical and grammatical meaning." Im Folgenden werden Frames ausschließlich im letztgenannten Sinne thematisiert. Als analytisches Hilfsmittel für korpuslinguistische Untersuchungen der Lexik spielen sie deswegen eine bedeutsame Rolle, weil Frame-Elemente bzw. Leerstellen als Annotationskategorien dienen können, mittels derer sich Belegstellen semantisch auswerten lassen. Frames ermöglichen es also, Bedeutungsprägungen zu identifizieren und zu untersuchen, ohne quasi an ihrer kognitiven Realität, vorbei zu theoretisieren".

6 Vgl. https://framenet.icsi.berkeley.edu. In vergleichbaren Frameworks, wie in Konerdings zunächst für lexikografische Zwecke entwickeltem Konzept der Matrixframes (Konerding 1993) und dessen kognitionslinguistischer Weiterentwicklung (Ziem 2008), sind es dagegen Prädikatorenklassen bzw. Leerstellen (Slots), die einen Frame strukturieren. In Barsalous psychologischem Zugriff, der dem Düsseldorfer Sonderforschungsbereich 991 „The Structure of Representations in Language, Cognition, and Science“ zugrunde liegt, werden vergleichbare Strukturen „Attribute" genannt (Barsalou 1993, 30-32; Löbner 2015, Abschnitt 3.4). 


\subsection{Framing und Medien-Frames: diskurssemantische Methodik}

In der Kommunikationswissenschaft werden Medien-Frames vorwiegend über mehr oder minder standardisierte, quantitative oder qualitativ vorgehende Medieninhaltsanalysen ermittelt. Der größte Teil dieser Studien bezieht sich dabei auf die vier von Entman $(1993,52)$ aufgestellten Dimensionen: Problemdefinition, Gründe, Lösungen und Bewertungen (vgl. Matthes 2009). Frames werden dabei entweder holistisch als vollständige Frames rekonstruiert oder aber ihre einzelnen Bestandteile werden zunächst kodiert und dann über statistische und/oder hermeneutische Verfahren zu Frames zusammengeführt (vgl. Matthes/Kohring 2008). Der hier vorgestellte Ansatz versteht im Gegensatz dazu Frames nicht als invariante Cluster einer begrenzten Zahl von Frame-Elementen, sondern als Strukturen, die datengeleitet rekonstruiert werden können und auf systematische Weise mit diskursiven Prozessen korrespondieren.

Zur diesbezüglichen diskurssemantischen Operationalisierung hat es sich als sinnvoll erwiesen, prinzipiell zwischen drei Strukturkonstituenten von Frames zu unterscheiden (vgl. Minsky 1975, 212; ausführlich: Ziem 2008, 283-366):

(a) Leerstelle / Frame-Element / Slot / Attribut: Je nach gewähltem frametheoretischen Ansatz wird mehr oder weniger explizit von „Slots“, „Leerstellen“ bzw. „Frame-Elementen“ gesprochen. Dabei handelt es sich um Konzeptualisierungsparameter, die ein aufgerufener Frame bereitstellt. Während es sich im Berkeleyer FrameNet bei Frame-Elementen um framespezifisch definierte semantische Rollen handelt, die auf der Basis systematischer Annotationen authentischer Sprachbelege (aus dem British National Corpus) ermittelt wurden, fällt Minskys Begriff des Slots (alternativ auch „terminal“ genannt) allgemeiner aus. Unabhängig von der Wahrnehmungsmodalität gilt hier ein Slot als ein spezifizierungsbedürftiger Parameter eines Schemas. Der Terminus „Attribut“ geht auf Barsalou (1993) zurück und wird im SFB 991 mit dem Konzepttyp des Funktionalbegriffes identifiziert (z. B. Löbner 2012).

(b) Filler / Wert / Instanz: „Fillers“, „Werte“ bzw. „Instanzen“ haben im semiotischen Sinne den Status von Token, die Leerstellen aufgrund aktual gegebener (Wahrnehmungs-)Daten konkretisieren. In FrameNet sind dies beispielsweise Phrasen, die syntaktisch von einem Zielausdruck abhängig sind und dementsprechend eine semantische Rolle spezifizieren. Im kognitionswissenschaftlichen Modell Barsalous handelt es sich allgemein um Spezifikationen von Attributen, deren 
semiotischer und ontologischer Status weitgehend offen bleibt (vgl. ausführlich: Busse 2012, 361-414).

(c) Standardwert / Default value: Dies sind schließlich Wissenselemente, die ähnlich wie „Fillers“ ebenfalls Leerstellen konkretisieren, ohne jedoch sprachlich oder anders realisiert zu sein. Sie müssen infolgedessen auf der Basis von Hintergrundwissen inferiert werden. Die Kategorie des Standardwertes ist in Minskys Konzeption angelegt und in Ziem (2008, 335-366) zum Zwecke der korpuslinguistischen Operationalisierung ausgearbeitet; sie spielt weder bei Barsalou (1993) und dessen Erweiterung im SFB 991 noch im FrameNet eine Rolle. ${ }^{7}$

In der Korpusanalyse in Abschnitt 3 greifen wir auf diese drei Strukturkonstituenten zurück. Mit Blick auf Möglichkeiten der korpusanalytischen Operationalisierung lassen sie sich wie folgt an (1) veranschaulichen.

(1) NSA collects vast amounts of data from telecom companies.

,Die NSA sammelt riesige Mengen an Daten von Telekom-Firmen.

Der Beispielsatz stammt aus der Berichterstattung der New York Times zur Snowden-Affäre. In diesem Fall entspricht die Nominalphrase NSA einem Wert der Leerstelle, die diejenige Instanz beschreibt, die etwas sammelt; traditionell handelt es sich dabei um die semantische Rolle des Agens. Eine weitere Leerstelle betrifft dasjenige, das gesammelt wird, hier konkretisiert durch die als direktes Objekt realisierte komplexe Nominalphrase vast amounts of data. Diese Leerstelle ist äquivalent mit der semantischen Rolle Thema (so im FrameNet) bzw. Affiziertes Objekt (nach von Polenz 2008, 170 ff.). Werden die semantischen Rollen, wie in FrameNet, framespezifisch definiert, können die Bezeichnungen variieren. FrameNet sieht hier etwa die semantische Rolle Individuen („Individuals“) vor. Schließlich gibt die Präpositionalphrase from telecom companies an, woher das, was gesammelt wird, stammt. In FrameNet findet sich hierfür die semantische Rolle Quelle („source“). Solche Instanzen der Frame-Elemente werden in FrameNet mit Indices semantisch annotiert, die die jeweils realisierte semantische Rolle des Frames angeben. Das so genannte „target“, also der frameevozierende Ausdruck, um dessen semantische Erfassung es geht, ist hier das Verb collect. Den

7 In FrameNet weist das Konzept der Null-Instantiierung eine gewisse Ähnlichkeit mit dem des Standardwertes auf; Null-Instantiierungen sind in FrameNet allerdings beschränkt auf sogenannte Kern-Frame-Elemente. 
gängigen Konventionen folgend, werden zur besseren Lesbarkeit semantische Rollen durch Kapitälchen sowie der frame-evozierende Ausdruck durch Fettdruck kenntlich gemacht (1).

(1) $\left[\mathrm{NSA}_{\mathrm{AgENT}}\right]$ collects [vast amounts of data InDIVDALS $_{\text {[ }}$ [from telecom companies SOURCE $_{\text {. }}$.

Insofern das Ziel darin besteht, Framing-Prozesse in Texten und Korpora aufzuspüren und im Detail zu beschreiben, ist es nötig, eine für einen Diskurs möglichst repräsentative Menge an Belegstellen zu annotieren und auszuwerten. Dazu eignen sich prinzipiell zwei Vorgehensweisen (vgl. die Detaildarstellungen in Scholz/Ziem 2015, 298-308): Ein induktiver Zugriff liegt vor, wenn die Leerstellen (bzw. Slots, Frame-Elemente) aus den Belegstellen heraus abgeleitet werden. Ein deduktives Verfahren geht dagegen von einem vorliegenden mehr oder weniger festen Set an Leerstellen aus, die als Annotationskategorien Einsatz finden. So können beispielsweise die im Berkeleyer FrameNet-Projekt eruierten Frame-Elemente verwendet werden. Der entscheidende Vorteil gegenüber der induktiven Methode besteht darin, auf empirisch validierte Frame-Elemente zurückgreifen zu können. Wird von ihnen ein Frame-Element nicht realisiert, so handelt es sich dabei um einen Befund, der einem induktiven Verfahren entgehen würde, da diesem zufolge Frame-Elemente allein auf der Basis des gegebenen sprachlichen Materials ermittelt werden. Gerade nicht realisierte Frame-Elemente können aber analytisch sehr interessant sein, insofern sie auf Wissensaspekte verweisen, die im Diskurs marginalisiert werden.

Das Ziel von FrameNet besteht darin, jedes Wort hinsichtlich jeder seiner lexikalischen Bedeutungen zu dokumentieren, indem seine semantische und syntaktische Valenz korpusbasiert erfasst und in einer Datenbank systematisch dokumentiert wird (vgl. den Überblick in Ziem 2014). Wie erwähnt, wird die Valenz framespezifisch in Form von semantischen Rollen angegeben. Anders als in traditionellen valenzgrammatischen Ansätzen im Anschluss an Tèsniere (1959) besteht hierbei die Grundannahme darin, dass nicht für jedes Wort (mindestens) ein Valenzrahmen anzugeben ist, sondern Frames vielmehr die kombinatorischen Eigenschaften einer Vielzahl bedeutungsähnlicher Ausdrücke, so genannter Lexikalischer Einheiten (LE), festlegen. Inzwischen umfasst die Datenbank ungefähr 
13.200 solcher LE und deckt damit grob den Grundwortschatz des Englischen ab. ${ }^{8}$ Der von collect aufgerufene Frame Gathering_up motiviert beispielsweise nicht nur die lexikalische Bedeutung von collect und collection, ${ }^{9}$ sondern auch von einer Vielzahl weiterer LE wie etwa die in FrameNet dokumentierten Lexeme amass.v, assemble.v, bring together.v, collect.v, convene.v, gather.v, herd.v und round up.v.

Es bleibt festzuhalten, dass sich die in der FrameNet-Datenbank erfassten Frames für die kommunikationswissenschaftliche Framing-Forschung nutzen lassen, indem Frame-Elemente (also valenzbedingte Leerstellen) zur Identifizierung von jenen Wissensaspekten (Werten) verwendet werden, die innerhalb eines Diskurszusammenhangs realisiert sind.

\section{Framing von Schlüsselwörtern in der medialen Berichterstattung zur Snowden-Affäre}

Ein linguistischer Zugang zu Framing-Prozessen ergibt sich aus der Anwendung des FrameNet zugrunde liegenden Valenz-Konzeptes auf diskurssemantische und zugleich kommunikationswissenschaftlich relevante Fragestellungen. So lassen sich die in einem Frame angelegten Frame-Elemente systematisch als Kategorien zur Annotation von syntaktisch realisierten semantischen Rollen nutzen. Dadurch, dass das Konzept der Valenz, d. h. der kombinatorischen Eigenschaften (in syntaktischer und semantischer Hinsicht) eines sprachlichen Ausdrucks, den konzeptionellen Ausgangspunkt von FrameNet bildet (vgl. Fillmore/Baker 2010, 321), werden in diesem Framework jene sprachlichen Einheiten annotiert, die in einer syntaktischen Abhängigkeit vom frame-evozierenden Zielausdruck stehen. Handelt es sich bei dem Zielausdruck um einen Valenzträger, legt dessen Valenzstruktur die relevanten Annotationseinheiten fest.

(2) [The federal authorities $]_{\text {AGENT }}[\text { routinely }]_{\text {MANNER }}$ collect $[$ data on phone calls] InDIVIDUALs.

,Die Landesbehörden sammeln routinemäßig Daten über Telefonanrufe.

8 Vgl. https://framenet.icsi.berkeley.edu/fndrupal/current_status; letzter Zugriff: 25. August 2015.

9 Wir folgen hier und im Folgenden der FrameNet-Konvention, die Frame-Bezeichnung durch die Verwendung des Schrifttyps Courier New erkennbar zu machen und Frame-Elemente in Kapitälchen zu setzen. 
In (2) sind beispielsweise vom Zielausdruck collect drei Einheiten syntaktisch abhängig, nämlich die Ergänzungen the federal authorities (realisiert in der grammatischen Funktion des Subjekts) und data on phone calls (realisiert als direktes Objekt) sowie die adverbiale Bestimmung routinely. Zusätzlich zur Annotation von Ergänzungen und Angaben werden in FrameNet auch Modifier wie z. B. adjektivische Attribute (etwa [vast] collection) und andere syntaktisch abhängige Einheiten wie Relativsätze (z. B. collection [that is stored electronically]) annotiert. ${ }^{10}$

Methodologisch vergleichbaren Studien folgend gliedert sich das im Folgenden angewendete Analyseverfahren in zwei Schritte (vgl. etwa Ziem/Scholz 2013; Scholz/Ziem 2015). Mithilfe einer Schlüsselwortanalyse sollen zunächst diskursiv zentrale LE sowie Indikatoren für lexikalische Bedeutungsprägungen identifiziert werden. Dabei können auch Kollokationsanalysen behilflich sein. In einem zweiten Schritt kommen Frames zum Einsatz, um die Schlüsselwörter semantisch im Detail zu analysieren. Beide Analyseverfahren sind korpuslinguistischer Natur, insofern die korpusgetriebene (corpus-driven) Schlüsselwortanalyse dazu dient, erste Forschungshypothesen zu generieren, während der eher korpusbasierten Frame-Analyse manuelle Annotationen zugrunde liegen, die eine feinkörnige Bedeutungsanalyse ermöglichen.

Das Untersuchungskorpus besteht aus Texten, die in der Online-Ausgabe der New York Times veröffentlicht wurden. Auswahleinheiten waren gemäß der Gliederung des Zeitungsauftritts Artikel, Blogbeiträge, Gastkommentare, Hintergrundanalysen, Leserbriefe und Rezensionen. Über die seiteneigene Suche im Online-Archiv wurden mittels der Begriffe „Snowden“ in Kombination mit „NSA“ oder „data“ insgesamt 322 Auswahleinheiten erhoben. Die Samplingperiode umfasst den Zeitraum vom 10. Juni 2013 (an diesem Tag wurde die erste Nachricht zu Edward Snowden im Guardian veröffentlicht) bis zum 2. August 2013, dem Tag, an dem Snowden vorläufig Asyl in Russland gewährt wurde und er den Transitbereich des Scheremetjewo-Flughafens in Moskau verließ.

\footnotetext{
${ }^{10}$ Auch sortale Nomen (wie Baum, Stein) werden in FrameNet erfasst, jedoch aufgrund ihrer fehlenden Valenz anders behandelt.
} 


\subsection{Schlüsselwortanalyse}

Schlüsselwörter sind LE, die in einem Korpus relativ zu einem Vergleichskorpus signifikant häufig auftreten. Der Grad der Signifikanz kann, je nach gewähltem Ansatz, mit statistischen Maßen wie Log-Likelihood oder Chi-Quadrat angegeben werden. Frequenz gilt dabei als Indikator für kognitive Verfestigung, sodass mit Blick auf die diskursive Relevanz einer LE angenommen werden kann, dass in dem Maße, wie ihre Auftretensfrequenz steigt, auch ihr Grad an kognitiver Präsenz wächst (Schmid 2010). Um die Wortfrequenz statistisch zu ermitteln, wurde die entsprechende Keyword List-Funktion der AntConc-Software (Version Windows 3.4.4) benutzt. ${ }^{11}$ Das Vergleichskorpus, das es erlaubt, die in einem gegebenen Korpus signifikant häufiger auftretenden LE zu ermitteln, wurde aus dem „Open American National Corpus“ bezogen (8.806 Dokumente). ${ }^{12}$ Die Schlüsselwortanalyse ergibt eine Liste mit mehr als 15.000 signifikant häufig vorkommenden LE (15.433), einige mit deutlichen thematischen Bezügen, wie etwa Snowden (Rangplatz 3; Log-Likelihood Wert 22276.794; Häufigkeit 3.261) oder surveillance (Rangplatz 33; Log-Likelihood Wert 4399.342; Häufigkeit 644). Problematisch und für das genutzte Online-Material spezifisch war indessen, dass dieses Vorgehen auch Funktionsbegriffe und Seitenbefehle des Webauftritts erfasste und diese als entsprechend relativ häufig auftretende LE listete.

Zusätzlich zur statistischen Auswertung wurde ein Teilkorpus bestehend aus zehn Texten (3,1\% des Gesamtkorpus) durchgesehen und qualitativ mit dem Ziel ausgewertet, die Analyse potenziell relevanter „Targets“, also frame-evozierender Ausdrücke, in ihrem diskursiven Ko-Text und Kontext zu erfassen. Dabei wurde deutlich, dass insbesondere Vorgänge des Sammelns von Daten und Informationen eine zentrale Rolle in der journalistischen Aufarbeitung und Beurteilung der Vorgänge spielen. Ausdruck findet dieser Fokus etwa in Phrasen wie „gathered online data" (Beitrag: „U.S. Confirms that it Gathers Online Data Overseas“; 6.6.2013), „collect data on phone calls“ (Beitrag: „President Obama’s Dragnet“; 6.6.2013) oder „Sweeping up records of telephone records“ (Beitrag: „Intelligence Chief Calls Leaks on U.S. Data Collection ,Reprehensible’“; 7.6.2013). In Kombi-

\footnotetext{
11 Vgl. http://www.laurenceanthony.net/software/antconc/; letzter Zugriff: 16. November 2015.

12 Andere Optionen wären etwa das „British National Corpus“ oder das „Brown Corpus of Present Day American English“; vgl. http://www.anc.org; letzter Zugriff: 16. November 2015.
} 
nation mit der signifikant höheren Auftretensfrequenz der sprachlichen Einheiten collection (Rangplatz 1.208; Log-Likelihood Wert 163.054; Häufigkeit 191; $\mathrm{p}<0.0001,0.01 \%$ ) und collect (Rangplatz 3.302; Log-Likelihood Wert 37.812; Häufigkeit $40 ; \mathrm{p}<0.0001,0.01 \%$ ) ist die diskursive Orientierung an Vorgängen des Ein- bzw. Ansammelns ein Indikator für den Grad ihrer diskursiven Relevanz. Collect bzw. collection sind mithin gute Kandidaten für öffentlich brisante Schlüsselwörter im Diskurs zur Snowden-Affäre.

\subsection{Semantische Annotationen mit FrameNet-Frames}

Der syntaktischen Einbettungsstruktur soll in der semantischen Analyse in zweierlei Hinsicht Rechnung getragen werden. Einerseits wird berücksichtigt, dass Wörter (hier: collect und collection) hinsichtlich ihrer semantischen und syntaktischen Valenz variieren, die sie in Texten selektiv ausschöpfen. Andererseits ist zu beachten, dass Wörter in Texten selbst in Gestalt unterschiedlicher semantischer Rollen auftreten können.

Die Analyse orientiert sich in konzeptioneller und methodischer Hinsicht an dem im FrameNet-Projekt praktizierten Vorgehen (vgl. die ausführliche Dokumentation in Ruppenhofer et al. 2010). Es wird davon ausgegangen, dass sich Bedeutungen von sprachlichen Ausdrücken, $d$. h. lexikalischen Einheiten und festen Mehrwortausdrücken, über Frames erfassen lassen. Ein sprachlicher Ausdruck evoziert demnach einen Frame, mit dem rekurrente, zum Teil kulturspezifische Erfahrungen und Ereignisse sowie die dazugehörigen Beteiligten und Situationsparameter analytisch zugänglich sind. Dies geschieht sprachlich in Gestalt von Frame-Elementen.

Das weitere analytische Vorgehen gliedert sich in drei Schritte. In einem ersten Schritt wird der zur framesemantischen Annotation der Schlüsselwörter dienliche Gathering_up-Frame erfasst. Der zweite Schritt betrifft die semantische Annotation der jeweils instantiierten Frame-Elemente des Gathering_upFrames. Dazu werden im Korpus alle Okkurrenzen der Zielausdrücke collect und collection identifiziert und die entsprechenden Belegstellen zu Konkordanzen für den Zweck der Annotation zusammengeführt. Schließlich folgt im Anschluss an die Datenannotation die quantitative und qualitative Auswertung der jeweils relevanten Frame-Elemente, um Aufschluss über dominante lexikalisch-semantische Konzeptualisierungen von collect bzw. collection zu geben. 
Im vorliegenden Fall ruft das Verb collect bzw. collection (wie eine Vielzahl anderer LE, so etwa die Verben amass.v, assemble.v, bring together.v, collect.v, convene.v, gather.v) den Gathering_up-Frame auf. ${ }^{13}$ Dieser beschreibt eine Handlung des Sammelns (etwa von Gegenständen) oder des Erfassens (z. B. von Daten), die sich mittels der in Tabelle 1 aufgelisteten und illustrierten Frame-Elemente genauer umreißen lässt. ${ }^{14}$

Die Bezeichnung der Frame-Elemente sind aus FrameNet übernommen und ins Deutsche übersetzt worden. Nicht enthalten in der Liste ist das Frame-Element DOMÄNE; uns erscheint es nicht gerechtfertigt zu sein, hierfür ein eigenes Frame-Element anzusetzen. Weder sind die in FrameNet annotierten Belege überzeugend, noch wird die Abgrenzung zum Frame-Element ART UND WEISE deutlich.

Weiterhin wird im Gathering_up-Frame unterschieden zwischen den Frame-Elementen INDIVIDUEN („Individuals“) und AGGREGAT („Aggregate“). Differenziert werden soll so zwischen den Objekten, die gesammelt werden, und der Sammlung (als komplexe Einheit), die dadurch entsteht. Diese Trennung hat sich in der empirischen Annotationspraxis insbesondere deshalb als unbrauchbar erwiesen, weil nur sehr selten eindeutig entschieden werden kann, welcher der beiden Fälle vorliegt. Erschwerend kommt hinzu, dass der Zielausdruck collection selbst das Aggregat zum Ausdruck bringt, eine Trennung zwischen den gesammelten Objekten und der Sammlung als Ergebnis des Sammelns also nicht sinnvoll möglich ist.

Der Gathering_up-Frame ist semantisch eng mit dem Aggregate-Frame verwandt. Anders als dieser enthält ersterer jedoch kein Frame-Element, das die Einheit spezifiziert, in der etwas gesammelt wird, bzw. die Person, der das Gesammelte gehört; im Aggregate-Frame übernimmt dies das Frame-Element CONTAINER_BESITZER. Im Zuge der Datenannotation hat sich erwiesen, dass dieses Frame-Element zwar konzeptionell relevant ist, jedoch nicht durch ein anderes

\footnotetext{
13 Vgl. https://framenet.icsi.berkeley.edu/fndrupal/index.php?q=frameIndex; letzter Zugriff 21. September 2015.

${ }^{14}$ Im Berkeleyer FrameNet-Projekt wird zwischen einem Kern- und einem Peripheriebereich von Frame-Elementen unterschieden. Die Differenzierung entspricht ungefähr der gängigen valenzgrammatischen Unterscheidung zwischen Ergänzungen und Angaben; periphere Elemente können geschlossen weggelassen werden. Im vorliegenden Fall gehören die Frame-Elemente AGENS, INDIVIDUEN und AGGREGAT und ÜBERGEORDNETES_EREIGNIS zum Kernbereich; alle anderen werden dem Peripheriebereich zugerechnet. Bei der folgenden diskurslinguistischen Auswertung kann diese valenztheoretisch motivierte Unterscheidung vernachlässigt werden.
} 
Frame-Element des Gathering_up-Frames vollständig abgedeckt wird. Aus diesem Grund haben wir CONTAINER_BESITZER als zusätzliche Annotationskategorie mit einbezogen.

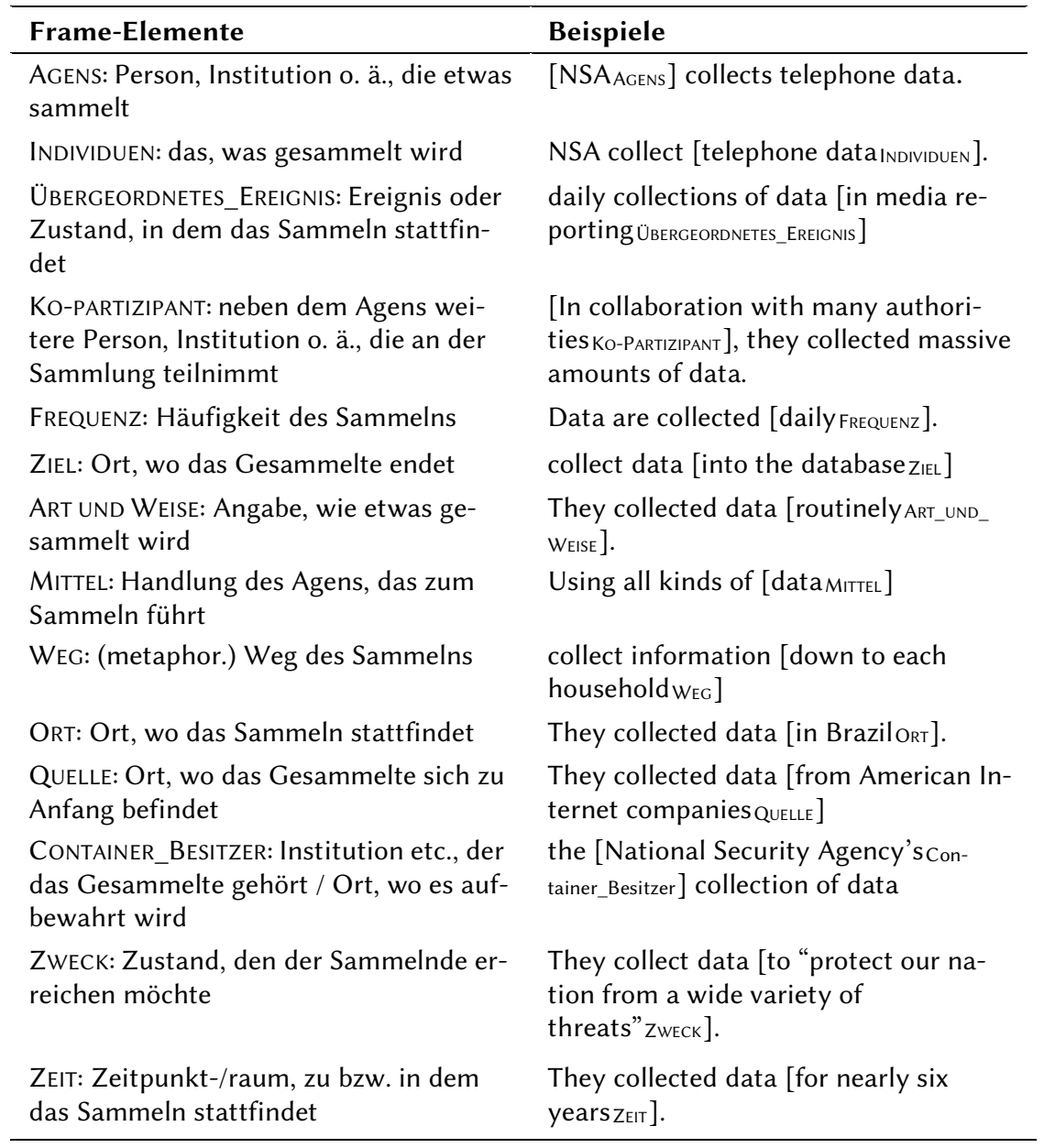

Tabelle 1: Frame-Elemente des Gathering_up-Frames (nach FrameNet)

Die Auswertung der annotierten Belegstellen gibt zum einen Aufschluss über diskursiv dominierende Bedeutungsaspekte (Frame-Elemente). Zum anderen dient 
sie als Werkzeug, um systematisch ausgeblendete oder marginalisierte Bedeutungsaspekte sichtbar zu machen sowie im Diskurs präsupponierte Wissenselemente zu identifizieren. Letztere werden in FrameNet durch so genannte NullInstanziierungen erfasst; dies sind verstehensnotwendige Frame-Elemente (also solche, die dem erwähnten Kernbereich zugerechnet werden), die aufgrund ihrer grammatischen Einbettung („konstruktionale Null-Instanziierung“) oder ihrer lexikalischen bzw. kontextuellen Realisierungsbedingungen („In/-Definite Null-Instanziierungen") nicht realisiert, aber in der Annotation mit erfasst werden (vgl. Ziem 2014, 282).

Nach der Identifizierung aller Okkurrenzen der Zielausdrücke * ${ }^{*}$ llect ${ }^{*}$ (also auch z. B. overcollected, collects oder collecting) und *collection* (etwa auch datacollection, collections) werden die insgesamt 284 Belegstellen aus dem Korpus zu Konkordanzen für den Zweck der Annotation zusammengeführt (Abb. 1).

\begin{abstract}
!en assuring Congress and the public that [the N.S.A.]AGT is not ${ }^{-}$collecting ese newly available procedures demonstrate an effort to avoid collecting There is no law barring any for-profit business from collecting is previous statement to Congress that [the agency]AGT did not collect at his statement in March to Congress that [N.S.A.]AGT was not collecting In fact, he said, the court found that [the N.S.A.]AGT had " overcollected siled logs of virtually all telephone calls in the United States and collected is, Mr. Bush rescinded his authorization to [the N.S.A.]AGT?? to collect ire," for legal purposes, the bulk communications when [it]AGT collected llk Internet metadata and gave [the agency]AGT a week to stop collecting [After]TIME?? [the contents]INDIV were collected The law under which [the government]AGT collected reason to believe that any but a tiny fraction [of the data]INDIV collected et infrastructure, Prism is further proof that [the agency]AGT is collecting nal intelligence certify that the purpose of the monitoring is to collect 'e electronic implants, and one involved the use of antennas to collect ng databases of "metadata" - [logs of all telephone calls] INDIV collected obscure passage in one of the Snowden documents - rules for collecting 'that when the agency's technicians probe for vulnerabilities to collect elligence, told a Senate committee that [the N.S.A.]AGT did not collect Clapper, the Director of National Intelligence, if [the N.S.A.]AGT collects nce Surveillance Court, showing that [the government]AGT was collecting in March. At that time Mr. Clapper denied that [the N.S.A.]AGT collected
\end{abstract}

[Americans' communications] INDIV [on a mass scale] [Americans' routine communications]INDIV. [just about every bit of data] INDIV they can about yo [records on millions of Americans] INDIV was untrue. [data on millions of America]INDIV was false.

"[information] [for technical reasons]??? and that the [the e-mail of foreigners] INDIV [from American Interı [bulk Internet metadata] INDIV and gave the agency a [them]INDIV, but instead only when human analysts : [it]INDIV and to block access to its existing database. [in that fashion]MANR, rules would be applied to scri [this data] INDIV, Section 215 of the Patriot Act, allow might possibly be suspicious.

[vast amounts of e-mails and other messages - incluc [foreign intelligence information about any non-Ame [transmissions] INDIV.

[from the major carriers] SOURCE and [similar data on [Internet data]INDIV that the Obama administration , [intelligence]INDIV, they also study foreign communi. [data on millions of Americans] INDIV.

['any type of data at all on millions or hundreds of $m$ [the phone records of most Americans] INDIV.

[private data on millions of American citizens] INDIV,

Abb. 1: Ausschnitt aus den annotierten Konkordanzen zu * collect* und * collection* 15

${ }^{15}$ Für die in Abb. 1 annotierten Realisierungen von Frame-Elementen werden folgende Abkürzungen verwendet: INDIV $=$ Individuum, AGT $=$ Agens, SOURCE $=$ Quelle, TIME $=$ Zeit, MANR $=$ Art_und_Weise; nicht ganz eindeutig zu treffende Zuschreibungen von Frame-Elementen werden mit Fragezeichen markiert. 


\subsection{Analyse und Interpretation der Annotationsergebnisse}

Ein wichtiges Ziel der Frame-Analyse besteht darin zu eruieren, inwiefern das Prädikationspotenzial des Zielausdrucks (hier: collect und collection) im jeweils avisierten diskursiven Zusammenhang selektiv ausgeschöpft wird. Das Prädikationspotenzial umfasst jene Wissensaspekte, die in sprachlicher Form dem Referenzobjekt prädikativ zugeschrieben werden. Sprachlich materialisiert sich das Prädikationspotenzial in Spezifizierungen von Frame-Elementen, also in syntaktisch unterschiedlich realisierten Informationseinheiten (etwa Phrasen in Argument- oder Adjunktfunktion), die vom Zielausdruck syntaktisch abhängig sind und dessen konzeptuellen Gehalt semantisch näher bestimmen.

\begin{tabular}{|l|c|c|c|c|}
\hline & \multicolumn{2}{|c|}{ Okkurrenzen (absolut) } & \multicolumn{2}{c|}{ Prozentualer Anteil } \\
\hline & $\begin{array}{c}\text { Snowden- } \\
\text { Affäre }\end{array}$ & $\begin{array}{c}\text { Vergleichskor- } \\
\text { pus }\end{array}$ & $\begin{array}{c}\text { Snowden- } \\
\text { Affäre }\end{array}$ & $\begin{array}{c}\text { Vergleichskor- } \\
\text { pus }\end{array}$ \\
\hline AGENS & 83 & 32 & 17,7 & 12,3 \\
\hline INDIVIDUEN & 253 & 81 & 54 & 31,2 \\
\hline ÜBERGEORDNETES_EREIGNIS & 2 & 4 & 0,4 & 1,5 \\
\hline KO-PARTIZIPANT & - & - & - & - \\
\hline FREQUENZ & - & 3 & - & 1,2 \\
\hline ZIEL & - & - & - & - \\
\hline INSTRUMENT & 10 & 7 & 2,1 & 2,7 \\
\hline ART_UND_WEISE & 57 & 53 & 12,2 & 20,5 \\
\hline MITTEL & - & - & - & - \\
\hline WEG & - & 1 & - & 0,4 \\
\hline ORT & 5 & 21 & 1 & 8,1 \\
\hline ZWECK & 10 & 13 & 2,1 & 4,5 \\
\hline QUELLE & 9 & 16 & 1,9 & 6,2 \\
\hline ZEIT & 33 & 17 & 1,3 & 4,6 \\
\hline CONTAINER_BESITZER & & 7,1 & 6,5 \\
\hline
\end{tabular}

Tabelle 2: Ergebnisse der Auswertung des Snowden-Korpus und des Vergleichskorpus 
Um zu prüfen, ob die ermittelten diskursiv dominierenden Bedeutungsaspekte, also Frame-Elemente, signifikant für den thematisch spezifischen Diskurs zu Snowden-Affäre sind, ist es erforderlich, an einem Vergleichskorpus des Standardsprachgebrauchs die dortige quantitative Verteilung der jeweils relevanten Frame-Elemente und der dominanten lexikalisch-semantischen Konzeptualisierungen von collect bzw. collection zu ermitteln und mit dem rekonstruierten Framing im Untersuchungskorpus zu vergleichen. Dazu wurden Okkurrenzen der Zielausdrücke * collect ${ }^{*}$ (also auch z. B. overcollected, collects oder collecting) und "collection" (etwa auch data-collection, collections) aus dem bereits für die Schlüsselwortsuche gebrauchten Open American National Corpus (8.806 Dokumente) identifiziert. Es wurden 216 zufällig ausgewählte Belegstellen (insgesamt 4.576 Nennungen der Schlüsselworte) zu Konkordanzen für den Zweck der Annotation zusammengeführt. Die Annotationsergebnisse sind in Tabelle 2 zusammengefasst.

Im direkten Vergleich fällt zunächst auf, dass die quantitative Verteilung sehr unterschiedlich ausfällt. Lediglich in einigen wenigen Fällen, nämlich den FE CONTAINER_BESITZER, INSTRUMENT und ÜBERGREIFENDES_INSTRUMENT, ist der prozentuale Anteil in einer vergleichbaren Marge. Besonders groß sind hingegen die Abweichungen bei QUELLE, ZWECK, ORT, ART_UND_WEISE und INDIVIDUEN. Während die ersten vier FE im Vergleichskorpus stärker dominieren als im Korpus zur Snowden-Affäre (dies allerdings im niederfrequenten Bereich meist deutlich unterhalb der 15 Prozent-Marke), gilt für das FE INDIVIDUEN umgekehrt, dass für sie eine starke Dominanz im Snowden-Korpus kennzeichnend ist. Die Unterschiede und Ähnlichkeiten der quantitativen Verteilung veranschaulicht das Balkendiagramm in Abbildung 2.

In der Snowden-Affäre liegt der diskursive Hauptfokus deutlich auf dem Objekt des Sammelns (INDIVIDUEN), weniger aber auf Snowden oder anderen Personen/Institutionen, die sammelnd tätig sind (AGENS), und überhaupt nicht auf den (technischen) Hilfsmitteln (INSTRUMENT) und die Art der Realisierung des Sammelns (ART_UND_WEISE).

Bei der Auswertung dieser Effekte medialen Framings ist zu berücksichtigen, dass im Korpus zur Snowden-Affäre das FE AGENS zwar knapp ein Drittel mal häufiger realisiert wird, jedoch über die im Vergleichskorpus annotierten (und in der Statistik erfassten) Instanzen hinaus AGENS-Realisierungen durch insgesamt 
31 Passivkonstruktionen unterdrückt werden. Somit wird der klare Fokus auf die sammelnde Instanz im Korpus zur Snowden-Affäre überdeutlich. Dass weder ZWECK noch QUELLE des Sammelns diskursiv in vergleichbarer Weise prominent sind wie im Vergleichskorpus, verstärkt den medialen Effekt der erzielten Rahmung der Einheit von Sammelnden (AGENS) und Gesammelten (INDIVIDUEN).

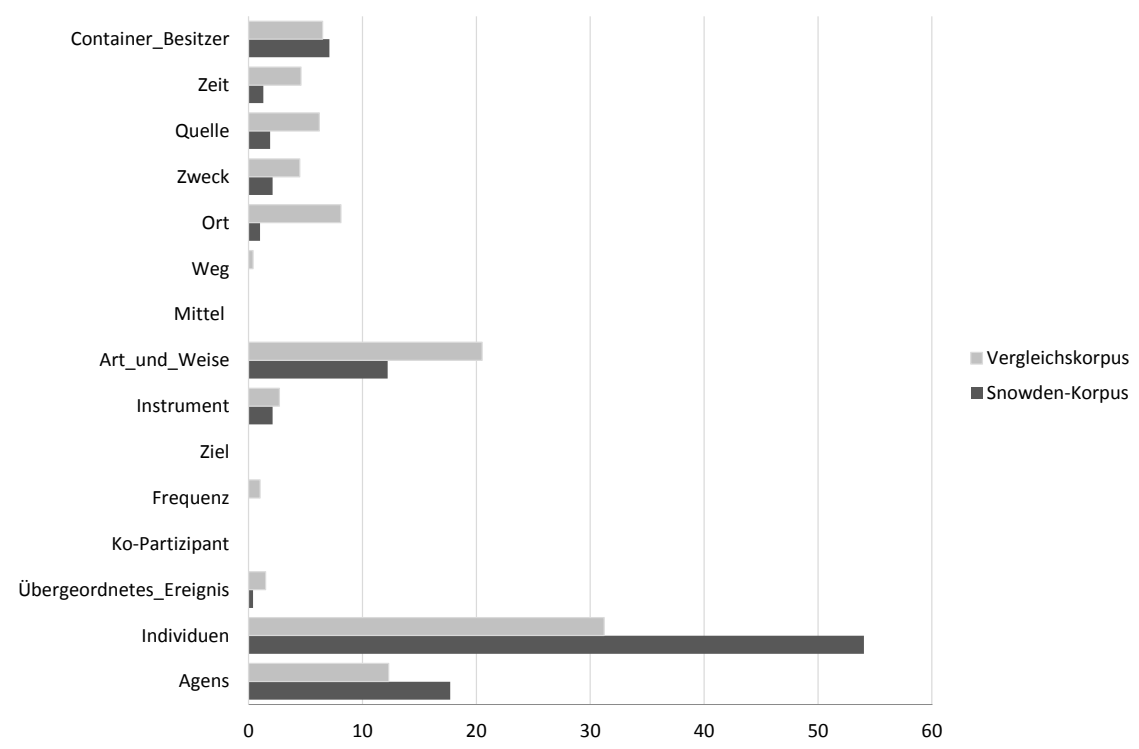

Abb. 2: Quantitative Verteilung der realisierten Frame-Elemente im Vergleich

In einer kommunikationswissenschaftlichen Interpretation dieser Befunde liegt es nahe, den festgestellten Fokus auf sammelnde Akteure (AGENS) und gesammelte Objekte (INDIVIDUEN) als Ausdruck einer journalistischen Framing-Strategie zu verstehen. In diesem Sinn liegt der Fokus der medialen Berichterstattung in der New York Times auf der bevorzugten Verbindung von datensammelnden Institutionen, allen voran der NSA, aber auch anderen staatlichen oder kommerziellen Organisationen wie dem britischen Geheimdienst bzw. den Betreibern von Telefonnetzen (z. B. Verizon) und Online-Plattformen (z. B. Facebook), und den durch sie gesammelten Daten (etwa Verbindungsdaten, Anschlussdaten etc.).

Es sind verschiedene Gründe für diese diskursstrategische Engführung auf eine sehr begrenzte Auswahl von Frame-Elementen denkbar, in der Analyse 
müssten sie jeweils im Detail plausibilisiert werden. Hinsichtlich der Abstraktheit und Vielschichtigkeit der aufgedeckten Sachverhalte kann das journalistische Framing etwa darum bemüht sein, Komplexität zu reduzieren und so den Blick auf die vermittel- und problematisierbare zentrale Verbindung von Sammelnden und Gesammeltem zu lenken. Der Fokus mag aber auch angesichts der sich tagesaktuell entwickelnden Affäre in der Vorläufigkeit der Informationen liegen, sodass die Etablierung eines stabilen Framing darauf abzielt, bereits identifizierbare Kernaspekte hervorzuheben, während unbelegte Behauptungen und Enthüllungen zunächst nicht bzw. erst in einem späteren und mithin nicht mehr durch das Korpus abgedeckten Zeitfenster Teil des Framing werden. Schließlich ist auch denkbar, dass der Befund nicht so sehr einem Diskurskalkül entspringt, sondern vielmehr dem unvollständigen, nur allmählich zugänglichen und von zunächst nur einer Quelle ausgehenden Informationsstand, nämlich den von Edward Snowden bereitgestellten Daten, geschuldet ist. In diesem Fall handelte es sich bei dem identifizierten medialen Framing gleichsam um ein Epiphänomen, das weder durch Diskursakteure motiviert noch durch sie bewusst gesteuert ist. Dieser letzten Erklärungsvariante steht aber der kommunikationswissenschaftliche Befund entgegen, dass sich gesellschaftliche, politische und publizistische Akteure in der Regel darum bemühen, Frames strategisch zu setzen und idealiter (massen-)medial auch durchzusetzen. So gesehen kann die eruierte Rahmung von datensammelnden Akteuren und gesammelten Daten interpretiert werden als Ausdruck und Ergebnis von „meaning work - the struggle over the production of mobilizing and countermobilizing ideas and meanings“" (Benford/Snow 2000: 613). Kurzum: Sie dient als argumentativer Ausgangspunkt dafür, Verantwortlichkeiten für den festgestellten Zustand zu benennen und Schlussfolgerungen darüber, was zu tun ist, abzuleiten.

\section{Schlussbemerkungen}

Wie öffentlich brisante Themen, Ereignisse oder Personen wahrgenommen werden, ist das Ergebnis eines mehrdimensionalen kommunikativen Prozesses, an dem eine Vielzahl an Faktoren konstitutiv beteiligt sind bzw. sein können. Dazu gehören nicht zuletzt die Massenmedien selbst, denn sie schaffen erst den Reso- 
nanzraum, in dem Brisanz in der Öffentlichkeit entstehen kann. Dieser Resonanzraum ist zumeist an Sprache gebunden, mit der Sachverhalte kommuniziert werden und in den öffentlichen Diskurs geraten. Der Sprachgebrauch - also die Wahl bestimmter sprachlicher Kategorien (Schlagwörter, Metaphern, grammatischer Konstruktionen, Textsorten usw.) - ist dabei niemals ,neutral', er trägt vielmehr zur begrifflichen Rahmung und mithin Erfassung des jeweiligen Bezugsobjektes bei.

Mediales Framing, das durch den Sprachgebrauch motiviert ist, stand im Mittelpunkt des vorliegenden Beitrages. Konkret untersucht wurden sprachlich gebundene Rahmungen am Beispiel der Schlüsselwörter collect und collection in der so genannten Snowden-Affäre. Empirische Grundlage bildeten dabei Belegstellen aus einem kompilierten Zeitungskorpus. Zur semantischen Annotation der Belegstellen dienten jene Frame-Elemente, die der von den Schlüsselwörtern aufgerufene Gathering_up-Frame bereitstellt.

In dreierlei Weise kann die framesemantisch motivierte Herleitung von FrameElementen und ihre Verwendung als Annotationskategorien genutzt werden, um die Analyse von Medienframes methodologisch und methodisch weiterzuentwickeln. Erstens trägt der vorgestellte Einbezug von semantischen Frames dazu bei, die Rekonstruktion von Framingprozessen in einem grundsätzlichen Konzept frame-basierter Wissens- und Verstehensprozesse zu verankern und linguistisch angemessen zu fundieren. Damit kann die linguistische Frame-Theorie (etwa im Anschluss an FrameNet) entschieden dazu beitragen, die kognitive und diskursive Dimension von Frames zu verknüpfen und in der empirischen Untersuchung nachzuweisen. Zweitens erlaubt die Aufstellung adaptierbarer Sets an Frame-Elementen die datengestützte und zugleich theoretisch begründete Elaborierung von Framestrukturen, statt ein allgemeingültiges, generisches und somit thematisch abstraktes Muster gebrauchen zu müssen. Drittens ist schließlich die so begründete Analyse prinzipiell offen für qualitative, am Verstehen orientierte Zugänge einerseits und quantifizierende Verfahren (korpuslinguistischer oder sozialwissenschaftlicher Provenienz), um Medien-Frames möglichst umfassend empirisch $\mathrm{zu}$ erfassen und zu validieren.

Die kommunikationswissenschaftliche Analyse von Framingprozessen kann ergänzend dazu beitragen, die framesemantischen Befunde als Teil strategischdiskursiver Aktivitäten zu verstehen. Diese Orientierung an Deutungskämpfen 
steht unter der Prämisse, dass Frames epistemisch keineswegs neutral sind, also Wissensbestände unabhängig von ihrer sozialen und kontextuellen Einbettung „repräsentieren“; vielmehr dienen sie dazu, Bewertungen vorzunehmen und zielen darauf ab, Zuständigkeiten, Gründe und Konsequenzen zu bestimmen und dominant zu setzen. In dieser Hinsicht geht die Analyse von Frames stets einher mit ihrer Verortung in gesellschaftlichen Problemfeldern, die sich mittels Frames als kulturellen Ressourcen konstituieren und kollektive Deutungsordnungen transformieren.

\section{Literatur}

Barsalou, Lawrence W. (1992): Frames, Concepts, and Conceptual fields. In: Lehrer, Adrienne / Kittay, Eva F. (eds.): Frames, Fields, and Contrasts. Hillsdale, NJ: Lawrence Erlbaum, 21-71.

Benford, Robert / David A. Snow (2000): Framing Processes and Social Movements: An Overview and Assessment. In: Annual Review of Sociology, 26, 611-639.

Boas, Hans C. (2005): Semantic Frames as Interlingual Representation for Multilingual Lexical Databases. In: International Journal of Lexicography, 18 (4), 445-478.

Busse, Dietrich (2012): Frame-Semantik. Ein Kompendium. Berlin / Boston: de Gruyter.

Carragee, Kevin M. / Roefs, Wim (2004): The Neglect of Power in Recent Framing Research. In: Journal of Communication, 54 (2), 214-233.

Chong, Dennis / Druckman, James N. (2007): Framing Theory. In: Annual Review of Political Science, 103-126.

Croft, William / Cruse, Alan D. (2007): Cognitive Linguistics. Cambridge: Cambridge University Press.

Dahinden, Urs (2006): Framing. Eine integrative Theorie der Massenkommunikation. Konstanz: UVK.

D’Angelo, Paul / Kuypers, Jim A. (eds.) (2009): Doing News Framing Analysis. Empirical and Theoretical Perspectives. London / New York: Routledge.

Entman, Robert (1993): Framing: Toward Clarification of a Fractured Paradigm. In: Journal of Communication, 43 (4), 51-58. 
Entman, Robert / Matthes, Jörg / Pellicano, Lynn (2009): Nature, Source and Effects of News Framing. In: Wahl-Jorgensen, Karin / Hanitzsch, Thomas (eds.): The Handbook of Journalism Studies. London / New York: Routledge, 175-190.

Fillmore, Charles J. (1977): Scenes-and-Frames Semantics. In: Zampolli, Antonio (eds.): Linguistic Structures Processing. Amsterdam: North Holland, 55-82.

Fillmore, Charles J. (1982): Frame Semantics. In: The Linguistic Society of Korea (ed.): Linguistics in the Morning Calm. Seoul: Hanshin, 111-137.

Fillmore, Chares J. (1985): Frames and the Semantics of Understanding. In: Quaderni di Semantica, 6 (2), 222-254.

Fillmore, Charles J. / Johnson, Christopher R. / Petruck, Miriam R. L. (2003): Background to FrameNet. In: International Journal of Lexicography, 16 (3), 235250.

Fillmore, Charles J. / Baker, Collin (2010): A frame approach to semantic analysis. In: Heine, Bernd / Narrog, Heiko (eds.): The Oxford Handbook of Linguistic Analysis. Oxford: Oxford University Press, 313-339.

Fraas, Claudia (1996): Gebrauchswandel und Bedeutungsvarianz in Textnetzen Die Konzepte IDENTITÄT und DEUTSCHE im Diskurs zur deutschen Einheit. Tübingen: Gunter Narr.

Fraas, Claudia / Pentzold, Christian (2011): Frames as Adaptive Networks of Meaning: A Frame-semantic Model for Communication Research. Vortrag auf der Annual Conference der International Communication Association, Boston (Section: Mass Communication).

Gamson, William A. / Modigliani, Andre (1989): Media Discourse and Public Opinion on Nuclear Power: A Constructionist Approach. In: American Journal of Sociology, 95 (1), 1-37.

Goffman, Erving (1977): Rahmen-Analyse: Ein Versuch über die Organisation von Alltagserfahrungen. Frankfurt a. M.: Suhrkamp.

Hermanns, Fritz (1995): Sprachgeschichte als Mentalitätsgeschichte. Überlegungen zu Sinn und Form und Gegenstand historischer Semantik. In: Gardt, Andreas / Mattheier, Klaus J. / Reichmann, Oskar (Hrsg.): Sprachgeschichte des Neuhochdeutschen. Gegenstände, Methoden, Theorien. Tübingen: Niemeyer, 69-101. 
Hertog, James / McLeod, Douglas M. (2001): A multiperspectival approach to framing analysis: A field guide. In: Reese, Steven / Gandy, Oscar / Grant, August (eds.): Framing Public Life. Mahwah, NJ: Lawrence Erlbaum, 139-161.

Holly, Werner (2001): ,Frame' als Werkzeug historisch-semantischer Textanalyse. Eine Debattenrede des Chemnitzer Paulskirchen-Abgeordneten Eisenstuck. In: Dieckmannshenke, Hajo / Meißner, Iris (Hrsg.): Politische Kommunikation im historischen Wandel. Tübingen: Stauffenberg, 125-146.

Johnston, Hank (2002): Verification and Proof in Frame and Discourse Analysis. In: Klandermans, Bert / Stagenborg, Suzanne (eds.): Methods of Social Movement Research. Minneapolis: University of Minnesota Press, 62-91.

Keller, Rudi (2003): Sprachwandel. Von der unsichtbaren Hand in der Sprache. 3. Auflage. Tübingen / Basel: Francke.

Klein, Josef (2002): Metapherntheorie und Frametheorie. In: Pohl, Inge (Hrsg.): Prozesse der Bedeutungskonstruktion. Frankfurt a. M. u. a.: Lang, 179-185.

Konerding, Klaus-Peter (1993): Frames und lexikalisches Bedeutungswissen. Untersuchungen zur linguistischen Grundlegung einer Frametheorie und zu ihrer Anwendung in der Lexikographie. Tübingen: Niemeyer.

Lakoff, George (1987): Women, Fire, and Dangerous Things: What categories reveal about the mind. Chicago: The University of Chicago Press.

Lakoff, George (2004): Don't think of an elephant! Know your values and frame the debate: the essential guide for progressives. Vermont: Chelsea Green.

Langacker, Ronald W. (1987): Foundations of Cognitive Grammar. Vol. 1: Theoretical Prerequisities. Stanford: Stanford University Press.

Liedtke, Frank / Wengeler, Martin / Böke, Karin (Hrsg.) (1991): Begriffe besetzen. Strategien des Sprachgebrauchs in der Politik. Opladen: Springer.

Löbner, Sebastian (2014): Evidence for frames from natural language. In: Gamerschlag, Thomas / Gerland, Doris / Petersen, Wiebke / Osswald, Rainer (eds.): Frames and Concept Types: Applications in Language and Philosophy. Heidelberg, New York: Springer, 23-68.

Löbner, Sebastian (2015): Functional Concepts and Frames. In: Gamerschlag, Thomas / Gerland, Doris / Osswald, Rainer / Petersen, Wiebke (eds.), Meaning, Frames, and Conceptual Representation (Studies in Language and Cognition 2). Düsseldorf: Düsseldorf University Press, 13-42. 
Lönnecker, Birte (2003): Konzeptframes und Relationen: Extraktion, Annotation und Analyse französischer Corpora aus dem World Wide Web. Berlin: Akademische Verlagsgesellschaft.

Lüders, Christian / Meuser, Michael (1997): Deutungsmusteranalyse. In: Hitzler, Ronald / Honer, Anne (Hrsg.): Sozialwissenschaftliche Hermeneutik. Opladen: Leske + Budrich, 57-79.

Matthes, Jörg (2007): Framing-Effekte. Zum Einfluss der Politikberichterstattung auf die Einstellung der Rezipienten. München: Fischer.

Matthes, Jörg (2009): What's in a Frame? A Content Analysis of Media-Framing Studies in the World's Leading Communication Journals, 1990-2005. In: Journalism and Mass Communication Quarterly, 86, 349-367.

Matthes, Jörg / Kohring, Matthias (2008): The Content Analysis of Media Frames: Toward Improving Reliability and Validity. In: Journal of Communication, 58 (2), 258-279.

Minsky, Marvin (1975): A Framework for Representing Knowledge In: Winston, P. H. (Hrsg.): The Psychology of Computer Vision. New York: Mc-Graw-Hill, 211-278.

Minsky, Marvin (1977): Frame-System Theory. In: Johnson-Leird, Philip / Wason, Peter C. (Hrsg.): Thinking: Readings in Cognitive Science. Cambridge: Cambridge University Press.

Minsky, Marvin (1988): The Society of Mind. New York: Simon \& Schuster.

Petersen, Wiebke (2007): Representation of Concepts as Frames. In: The Baltic International Yearbook of Cognition, Logic and Communication 2, 151-170.

Plaß, Christine / Schetsche, Michael (2001): Grundzüge einer wissenssoziologischen Theorie sozialer Deutungsmuster. In: Sozialer Sinn 3, 511-536.

Polenz, Peter von (2008): Satzsemantik. Grundbegriffe des Zwischen-den-ZeilenLesens. Berlin / New York: de Gruyter.

Ruppenhofer, Josef et al. (2010): FrameNet II: Extended Theory and Practice. University of California at Berkeley. Online: http://framenet.icsi. berkeley.edu/ book/book.html [14.11.2015].

Schank, Roger C. / Abelson, Robert P. (1977): Scripts, Plans, Goals and Understanding: An Inquiry into Human Knowledge Structures. Hillsdale, NJ: Lawrence Erlbaum. 
Scheufele, Dietram A. (1999): Framing as a Theory of Media Effects. In: Journal of Communication, 49 (1), 103-122.

Scheufele, Bertram (2003): Frames - Framing - Framing-Effekte. Theoretische und methodische Grundlegung des Framing-Ansatzes sowie empirische Befunde zur Nachrichtenproduktion. Wiesbaden: Westdeutscher Verlag.

Schmid, Hans-Jörg (2010): Does frequency in text really instantiate entrenchment in the cognitive system? In: Glynn, Dylan / Fischer, Kerstin (eds.). Quantitative methods in cognitive semantics: Corpus-driven approaches. Berlin / New York: de Gruyter, 101-133.

Scholz, Ronny / Ziem, Alexander (2013): Lexikometrie meets FrameNet: das Vokabular der „Arbeitsmarktkrise“ und der „Agenda 2010“ im Wandel. In: Wengeler, Martin / Ziem, Alexander (Hrsg.): Sprachliche Konstruktionen von Krisen: Interdisziplinäre Perspektiven auf ein fortwährend aktuelles Phänomen. Bremen: Hempen, 155-184.

Simon, Adam F. / Jerit, Jennifer (2007): Toward a Theory Relating Political Discourse, Media, and Public Opinion. In: Journal of Communication 57, 254-271.

Stötzel, Georg (1980): Konkurrierender Sprachgebrauch in der deutschen Presse. Sprachwissenschaftliche Textinterpretation zum Verhältnis von Sprachbewusstsein und Gegenstandskonstitution. In: Wirkendes Wort 30, 39-53.

Stötzel, Georg (1995): Einleitung. In: Stötzel, Georg / Wengeler, Martin (Hrsg.): Kontroverse Begriffe. Geschichte des öffentlichen Sprachgebrauchs in der Bundesrepublik Deutschland. Berlin / New York: de Gruyter, 1-17.

Tannen, Deborah (1993): Framing in Discourse. New York: Oxford University Press.

Tankard, John W. (2001): An Empirical Approach to the Study of Media Framing. In: Reese, Steven / Gandy, Oscar / Grant, Augustus (eds.): Framing Public Life. Mahwah, NJ: Lawrence Erlbaum, 95-106.

Tèsniere, Lucien (1959): Elements de Syntaxe Structurale. Paris: Klingksieck. Van Gorp, Baldwin (2009): Strategies to Take Subjectivity Out of Framing Analysis. In: D’Angelo, Paul / Kuypers, Jim A. (eds.): Doing News Framing Analysis. Empirical and Theoretical Perspectives. London / New York: Routledge, 84-109. 
Wengeler, Martin (1996): Sprachthematisierungen in argumentativer Funktion. Eine Typologie. In: Böke, Karin / Jung, Matthias / Wengeler, Martin (Hrsg.): Öffentlicher Sprachgebrauch. Opladen: Westdeutscher Verlag, 413-430.

Ziem, Alexander (2008): Frames und sprachliches Wissen. Kognitive Aspekte der semantischen Kompetenz. Berlin / New York: de Gruyter.

Ziem, Alexander (2013): Frames als Prädikations- und Medienrahmen: auf dem Weg zu einem integrativen Ansatz? In: Fraas, Claudia / Meier, Stefan / Pentzold, Christian (Hrsg.): Online-Diskurse. Theorien und Methoden transmedialer Online-Diskursforschung “. Köln: Halem, 136-172.

Ziem, Alexander (2014): Von der Kasusgrammatik zum FrameNet: Frames, Konstruktionen und die Idee eines Konstruktikons. In: Ziem, Alexander / Lasch, Alexander (Hrsg.): Grammatik als Inventar von Konstruktionen? Sprachwissen im Fokus in der Konstruktionsgrammatik. Berlin / New York: de Gruyter, 263-290.

Ziem, Alexander (2017): Lexik - korpusanalytisch. In: Niehr, Thomas / Kilian, Jörg / Wengeler, Martin (Hrsg.): Handbuch Sprache und Politik. Bremen: Hempen, 169-194.

Ziem, Alexander / Scholz, Ronny (2013): Das Vokabular im diskurshistorischen Vergleich: Skizze einer korpuslinguistischen Untersuchungsheuristik. In: Kämper, Heidrun / Warnke, Ingo (Hrsg.): Diskurs interdisziplinär. Berlin / Boston: de Gruyter, 281-313. 